\title{
Anestesia epidural com ropivacaína, lidocaína ou associação de lidocaína e xilazina em cães. Efeitos cardiorrespiratório e analgésico
}

\author{
Epidural anaesthesia using ropivacaine, lidocaine or the combination of lidocaine and xylazine in dogs. \\ Cardiorespiratory and analgesic effects
}

\author{
Simone Salata Gasparini ${ }^{\mathrm{I}}$ Stelio Pacca Loureiro Luna*I ${ }^{*}$ Renata Navarro Cassu ${ }^{\text {III }}$ Edilson Uiechi ${ }^{\mathrm{I}}$ \\ Adalberto José Crocci ${ }^{\mathrm{IV}}$
}

\section{RESUMO}

Este estudo investigou o efeito da ropivacaína, da lidocaína e da associação de lidocaína e xilazina na anestesia epidural de cães. Trinta cães foram tranqüilizados com acepromazina intravenosa, distribuídos em três grupos e submetidos à anestesia epidural no espaço lombo-sacro, com lidocaína $2 \%$ com vasoconstrictor (GL), ropivacaína 1\% (GR) ou com xilazina associada à lidocaína (GXL). Mensuraram-se as freqüências cardíaca (FC) e respiratória (f), a pressão arterial sistólica (PAS), a concentração final expirada de $\mathrm{CO}_{2}\left(\mathrm{EtCO}_{2}\right)$, $o$ volume minuto (VM) e a temperatura retal (T). Para avaliação da analgesia somática, utilizou-se o teste do panículo e o teste térmico a $55^{\circ} \mathrm{C}$. Os protocolos produziram anestesia da região retro-umbilical, sendo que a associação XL produziu bloqueio anestésico mais cranial, porém causou bradicardia moderada. A duração da anestesia foi mais prolongada nos animais dos grupos GXL (240 min) e GR (250 min), quando comparada as do grupo GL (120 min).

Palavras-chave: lidocaína, xilazina, ropivacaína, epidural, cão.

\section{ABSTRACT}

This study was aimed at investigating the effects of ropivacaine, lidocaine or lidocaine combined with xylazine for epidural anaesthesia in dogs. Thirty dogs were sedated with acepromazine IV, divided in to three groups and submitted to lumbosacral epidural anaesthesia using $2 \%$ lidocaine with adrenaline $(L)$ or $1 \%$ ropivacaine $(R)$ or xylazine combined with lidocaine $(X L)$. Heart and respiratory rates, systolic arterial blood pressure, $\mathrm{EtCO}_{2}$, minute volume and temperature were measured. Cutaneous anaesthesia was investigated using a forceps and thermic stimulus. All protocols produced retroumbilical anaesthesia. The combination of XL produced a more cranial anaesthetic block, with moderate bradycardia. The duration of the anaesthesia was more prolonged in animals treated with XL (240min) and $R$ (250min), when compared to L alone (120min).

Key words: lidocaine, xylazine, ropivacaine, epidural, dog.

\section{INTRODUÇÃO}

Apesar de ser uma técnica antiga, a anestesia epidural tem sido usada, freqüentemente, pela possibilidade do emprego de doses de fármacos menores que as administradas por outras vias, com menores efeitos colaterais, além de promover analgesia intra e pós-operatória (MCMURPHY, 1993) e permitir a redução da resposta de estresse pós-cirúrgico (WEISSMAN, 1990).

O desenvolvimento de técnicas anestésicas seguras, simples e de baixo custo, que viabilizem a realização de ovário-salpingo-histerectomia (OSH), em ocasiões em que não haja disponibilidade de equipamentos de anestesia inalatória, é um constante desafio, particularmente, em situações de carência econômica ou em campanhas de controle populacional. Com relação à cesárea, a anestesia epidural é a técnica

\footnotetext{
${ }^{\text {I} F a c u l d a d e ~ d e ~ M e d i c i n a ~ V e t e r i n a ́ r i a ~ e ~ Z o o t e c n i a ~(F M V Z), ~ U n i v e r s i d a d e ~ E s t a d u a l ~ P a u l i s t a ~(U n e s p), ~ B o t u c a t u, ~ S P, ~ B r a s i l . ~}$

IIDepartamento de Cirurgia e Anestesiologia Veterinária, Unesp, Campus de Botucatu, 18618-000, Rubião Junior, Botucatu, SP, Brasil. Email: stelio@fmvz.unesp.br. Autor para correspondência.

IIIDepartamento de Cirurgia e Anestesiologia Veterinária, Faculdade de Ciências Agrárias, Universidade do Oeste Paulista (Unoeste). Rodovia Raposo Tavares, Km 572, Campus II, Bairro Limoeiro, 19067-175, Presidente Prudente, SP, Brasil. E-mail: renavarro@uol.com.br.

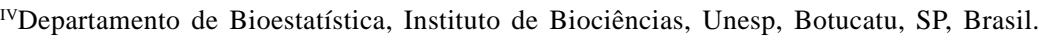


mais segura, tanto do ponto de vista materno como neonatal (LUNA et al., 2004).

Entre as vantagens da anestesia epidural sobre a anestesia geral, tem-se a segurança, por minimizar as alterações cardiorrespiratórias, destacando-se a fácil execução da técnica e o custo acessível (MASSONE, 2003). Ademais, o fármaco administrado por esta via sofre menor absorção e, portanto, acarreta efeitos sistêmicos menos pronunciados (SKARDA, 1987).

O uso isolado de anestésico local, normalmente da lidocaína, não é suficiente para produzir anestesia que viabilize a OSH em cães (ISHIY et al., 2002), já que os ovários são inervados pelo $3^{\circ}$ e $4^{\circ}$ nervos lombares (BAILEY et al., 1988). Adicionalmente, a lidocaína é um fármaco de curta ação, sendo limitada em cirurgias prolongadas, havendo necessidade da associação com fármacos de longa duração (CRUZ et al., 1997; ISHIY et al., 2002). A ropivacaína, agente de longa duração e homóloga à bupivacaína, promove bloqueio sensitivo de duração igual ou discretamente inferior, com período de bloqueio motor e toxicidade nervosa e cardíaca menores que os daquele fármaco (WOLFF et al., 1995; FELDMAN et al., 1996).

Objetivou-se avaliar uma técnica de anestesia epidural que viabilizasse bloqueio sensitivo e motor prolongado além da possibilidade de dessensibilização da região abdominal cranial, visando a atingir o nervo gênito-femoral, responsável pela inervação dos ovários. Para tal, investigaram-se os efeitos cardiorrespiratórios e analgésico somático produzidos pela lidocaína, pela ropivacaína ou pela lidocaína associada à xilazina no espaço epidural de cães.

\section{MATERIAL E MÉTODOS}

Foram empregados trinta cães, machos e fêmeas, sem raça definida, adultos, com peso médio corpóreo de $12 \pm 3 \mathrm{~kg}$, provenientes do canil do Biotério Central da Unesp, Campus de Botucatu. Todos os animais foram submetidos a jejum alimentar e hídrico de 12 e 2 horas, respectivamente. A medicação préanestésica (MPA) para todos os grupos foi realizada com $0,1 \mathrm{mg} \mathrm{kg}^{-1}$ de acepromazina ${ }^{\mathrm{a}}$, por via intravenosa, pela veia cefálica. A anestesia epidural foi realizada 15 minutos após a MPA. Um botão anestésico foi feito no local da injeção, localizado por palpação das tuberosidades ilíacas e da crista dorsal de L7, com o animal em decúbito esternal, estendendo-se os membros pélvicos, cranialmente (CRUZ et al., 1997). Uma agulha 40x8 foi introduzida cranialmente em ângulo aproximado de 70 (entre L7-S1), até ultrapassar o ligamento amarelo e atingir o espaço epidural, o qual foi confirmado pela aspiração de uma gota do anestésico, depositada no canhão da agulha.

Os animais foram distribuídos aleatória e equitativamente em três grupos, e cada grupo de 10 animais foi submetido à anestesia epidural com lidocaína $2 \%^{\mathrm{b}}\left(5 \mathrm{mg} \mathrm{kg}^{-1}\right.$, equivalente a $\left.1 \mathrm{ml}^{4} \mathrm{~kg}^{-1}\right)(\mathrm{GL})$; ropivacaína $1 \%{ }^{\mathrm{c}}\left(2,51 \mathrm{mg} \mathrm{kg}^{-1}\right.$ equivalente a $\left.1 \mathrm{ml} 4 \mathrm{~kg}^{-1}\right)$ (GR) e xilazina $2 \%{ }^{\mathrm{d}}\left(0,251 \mathrm{mg} \mathrm{kg}^{-1}\right)$ diluída em lidocaína $2 \%$ (4,751 $\left.\mathrm{mg} \mathrm{kg}^{-1}\right)$, de modo a perfazer um volume total de $1 \mathrm{ml} 4 \mathrm{~kg}^{-1}$ (GXL). A injeção dos fármacos foi realizada em período aproximado de 45 a 60 segundos, com velocidade constante. Foram avaliados: freqüência cardíaca (FC), com estetoscópio e monitor cardíaco ${ }^{\mathrm{e}}$ com os eletrodos fixados com adesivos, cuja leitura foi realizada na segunda derivação de Eithoven; freqüência respiratória (f), mensurada pela observação dos movimentos respiratórios em um minuto; pressão arterial sistólica (PAS), mediante monitor de pressão arterial não-invasivof ${ }^{\mathrm{f}}$, pela adaptação do manguito pediátrico na região proximal do rádio, respeitando-se uma relação de 0,4 entre a largura do manguito e o comprimento da circunferência do membro; concentração de $\mathrm{CO}_{2}$ no final da expiração $\left(\mathrm{ETCO}_{2}\right)$, mensurada utilizando-se capnógrafo $^{g}$ de aspiração, cujo tubo coletor foi colocado na narina; volume-minuto (VM), mensurado com ventilômetro ${ }^{\mathrm{h}}$, por meio de máscara acoplada à face e temperatura retal (T), com termômetro clínico digitali. Os parâmetros foram mensurados 15 minutos após a aplicação da MPA, e a cada 15 minutos após a anestesia epidural, até o retorno do reflexo interdigital e de movimento espontâneo do membro pélvico. Foram determinados também: o tempo entre a injeção epidural até o relaxamento do esfíncter anal; o tempo até a perda do reflexo interdigital (período de latência), aferido por meio de estímulo nociceptivo mecânico, com pinça hemostática Kelly (RECTOR et al., 1997), até a ausência de reflexo interdigital em ambos os membros pélvicos. A partir daí, a anestesia foi avaliada com estímulos nociceptivos somáticos, aplicados a cada 15 minutos, imediatamente após a mensuração das variáveis paramétricas. A analgesia somática foi avaliada na região dorsal do animal, pelo teste do panículo, com pinça Kelly, e o estímulo térmico com bastão de metal aquecido em banho-maria até $55^{\circ} \mathrm{C}$, colocando-o sobre a pele, previamente tricotomizada, por um tempo máximo de 15 segundos, simulando o teste de placa quente em ratos (MAZZANTI \& BRAGHIROLI, 1994; SAHNI et al., 1995). Os estímulos foram aplicados na região dorsal, iniciando-se na área lombo-sacra, em direção cranial às vértebras lombares e torácicas, até ser identificada a resposta positiva do animal perante o estímulo nociceptivo. Essa avaliação permitiu a identificação da 
extensão do bloqueio anestésico, uma vez que os estímulos nociceptivos foram realizados em áreas anatômicas específicas. Dessa forma, o estímulo foi aplicado em cada uma das vértebras, sendo registrado o ponto na coluna vertebral em que o animal apresentava sensibilidade. Para a avaliação dos dados, foi realizado o somatório do número de vértebras dessensibilizadas, a partir do espaço lombo-sacro. Os animais que manifestavam resposta positiva perante o estímulo nociceptivo somático levantavam a cabeça, virando a face em direção à área que estava sendo avaliada, demonstrando resistência a esse estímulo, tentavam morder o bastão metálico ou pinça e emitiam gemidos ou latidos durante a avaliação.

Foram utilizados testes estatísticos nãoparamétricos, com os resultados dados pelas medianas e pelas amplitudes semi-quartílicas, visando a comparar os momentos dentro de cada grupo pelo teste de Friedman, e grupos em cada momento pelo teste de Kruskal-Wallis. Para avaliação da extensão do bloqueio anestésico, foi realizada uma contagem do número médio de vértebras dessensibilizadas, com transformação dos valores originais para ( $\mathrm{x}$, sendo utilizada a análise de variância com um fator (ANOVA), para comparação, em média, dos grupos em cada momento avaliado. As comparações entre médias foram verificadas pelo teste de Tukey. Para as variáveis tempo de relaxamento do esfíncter anal, perda do reflexo interdigital, recuperação deste reflexo e estação, os grupos foram comparados pelo teste de Kruskal-Wallis. Todos os testes foram realizados em nível de significância de 5\%.

\section{RESULTADOS}

A anestesia epidural foi realizada com sucesso em todos os animais, sendo que não foi observada nenhuma intercorrência durante a sua execução. Com relação ao sistema cardiovascular, nos animais do GXL, a FC permaneceu inferior aos valores do GL ao longo do tempo, porém somente dois animais tratados com xilazina/lidocaína apresentaram bradiarritmia (FC $<72$ bat $\mathrm{min}^{-1}$ ). Os animais do GL apresentaram maiores valores de freqüência cardíaca aos 30 e dos 60 aos 120 minutos, em comparação ao GR. Não houve diferença entre o GXL e o GR para esta variável. Não foi observada diferença significativa para PAS, entre momentos ou entre grupos. Mínimas alterações respiratórias foram observadas ao longo do tempo e entre os grupos. Houve redução da temperatura no GL, superior em relação aos demais grupos ao longo do tempo, diferença representada nas equações de regressão (Tabela 1). Houve alteração comportamental evidente em todos os animais do GXL, os quais apresentaram sinais de sedação, mantendo-se com o globo ocular rotacionado e ficando sonolentos. Os tempos entre a administração da anestesia epidural até o relaxamento do esfíncter anal e a perda do reflexo interdigital foram maiores no GR em relação aos demais grupos. Os tempos observados até o retorno do reflexo interdigital e a posição quadrupedal foram superiores para o GXL e GR em comparação ao GL (Tabela 2). A altura do bloqueio sensitivo obtido no GXL foi mais cranial em relação aos demais grupos (Tabelas 2 e 3).

\section{DISCUSSÃO}

A redução da freqüência cardíaca, em relação aos valores basais, observada no GXL, provavelmente, está relacionada ao efeito $(\alpha 2$ adrenoceptor agonista da xilazina, produzindo bradicardia, que pode culminar em BAV (GROSS, 2003). Em estudo semelhante, KELAWALA et al. (1996) relataram decréscimo das freqüências cardíaca e respiratória e da pressão arterial média após administração epidural de xilazina em cães. Apesar da xilazina via epidural apresentar efeitos sistêmicos menos evidentes que por via parenteral (VIEIRA et al., 1993), neste estudo, assim como nos relatos em outras espécies, observou-se que a aplicação epidural desse fármaco também determinou efeitos depressores no sistema cardiovascular. Os efeitos sistêmicos devemse, possivelmente, à absorção do fármaco pela circulação. Nesta pesquisa, embora tenha sido observada redução da freqüência cardíaca, alterações sistêmicas mais graves, inerentes aos efeitos dos fármacos $\alpha_{2}$-agonistas, como arritmias e BAV, não foram identificadas, não sendo necessária intervenção farmacológica, corroborando prévios resultados, em que a administração epidural de $0,25 \mathrm{mg} \mathrm{kg}^{-1}$ de xilazina diminuiu a resposta hemodinâmica, porém não causou efeitos adversos relevantes (RECTOR et al., 1997). Deve-se salientar que a bradicardia pode representar efeito colateral importante em animais debilitados, apesar da ausência de hipotensão neste experimento. A lidocaína, especialmente, quando associada à adrenalina, apresenta lenta absorção sistêmica, determinando boa margem de segurança para o sistema cardiovascular, bem como a ropivacaína (WOLFF et al., 1995). Neste estudo, os grupos tratados com esses fármacos não apresentaram alterações importantes, no concernente à frequiência cardíaca ou à pressão arterial sistólica. No entanto, os animais anestesiados com ropivacaína apresentaram discreta redução da freqüência cardíaca. No homem, observou-se bradicardia e hipotensão, como possíveis efeitos 
Tabela 1 - Mediana e amplitude semiquartílica dos valores de freqüência cardíaca (FC), pressão arterial sistólica (PAS), concentração de CO no final da expiração $\left(\mathrm{ETCO}_{2}\right)$, volume minuto $(\mathrm{VM})$ e freqüência respiratória (f). Valores médios e amplitude semi-quartílica de temperatura retal $(\mathrm{T})$. Coeficiente de regressão da temperatura retal em cães submetidos à tranqüilização com acepromazina e à anestesia epidural com lidocaína $2 \%$ com vasoconstritor (GL), xilazina $2 \%$ associada à lidocaína (GXL) ou ropivacaína 1\% (GR).

\begin{tabular}{|c|c|c|c|c|c|c|c|c|c|}
\hline & MPA & $15 \mathrm{~min}$ & $30 \mathrm{~min}$ & $45 \mathrm{~min}$ & 60min & $75 \mathrm{~min}$ & 90min & $105 \mathrm{~min}$ & $120 \mathrm{~min}$ \\
\hline \multicolumn{10}{|c|}{ FC (bat/min) } \\
\hline GL & $102 \pm 12$ & $96 \pm 12^{\mathrm{a}}$ & $98 \pm 6^{\mathrm{a}}$ & $96 \pm 17^{\mathrm{a}}$ & $102 \pm 21^{\mathrm{a}}$ & $98 \pm 20^{\mathrm{a}}$ & $95 \pm 20^{\mathrm{a}}$ & $100 \pm 12^{\mathrm{a}}$ & $104 \pm 17^{\mathrm{a}}$ \\
\hline GXL & $98 \pm 14^{\mathrm{A}}$ & $73 \pm 12^{\mathrm{bB}}$ & $72 \pm 9^{\mathrm{bB}}$ & $70 \pm 10^{\mathrm{bB}}$ & $64 \pm 9^{\mathrm{bB}}$ & $72 \pm 10^{\mathrm{bB}}$ & $77 \pm 8^{\mathrm{bB}}$ & $75 \pm 7^{\mathrm{bB}}$ & $74 \pm 8^{\mathrm{bB}}$ \\
\hline GR & $94 \pm 14^{\mathrm{A}}$ & $84 \pm 15^{\mathrm{abAB}}$ & $80 \pm 13^{\mathrm{bAB}}$ & $87 \pm 18^{\mathrm{abAB}}$ & $76 \pm 18^{\mathrm{bAB}}$ & $80 \pm 12^{\mathrm{bAB}}$ & $80 \pm 5^{\mathrm{bAB}}$ & $81 \pm 11^{\mathrm{bAB}}$ & $85 \pm 14^{\mathrm{bAB}}$ \\
\hline \multicolumn{10}{|c|}{ PAS (mmHg) } \\
\hline GL & $115 \pm 7$ & $104 \pm 10$ & $109 \pm 12$ & $110 \pm 12$ & $110 \pm 14$ & $112 \pm 5$ & $108 \pm 6$ & $110 \pm 5$ & $120 \pm 12$ \\
\hline GXL & $117 \pm 8$ & $115 \pm 8$ & $118 \pm 14$ & $117 \pm 8$ & $120 \pm 6$ & $120 \pm 12$ & $122 \pm 4$ & $118 \pm 7$ & $110 \pm 17$ \\
\hline GR & $120 \pm 5$ & $111 \pm 5$ & $111 \pm 6$ & $118 \pm 11$ & $118 \pm 10$ & $118 \pm 18$ & $117 \pm 12$ & $120 \pm 13$ & $114 \pm 10$ \\
\hline \multicolumn{10}{|c|}{$\mathrm{ETCO}_{2}(\mathrm{mmHg})$} \\
\hline GL & $35 \pm 2$ & $36 \pm 3$ & $35 \pm 2$ & $35 \pm 2$ & $36 \pm 3$ & $36 \pm 2$ & $36 \pm 3$ & $37 \pm 2$ & $37 \pm 2$ \\
\hline GXL & $35 \pm 4$ & $34 \pm 3$ & $34 \pm 3$ & $32 \pm 3$ & $33 \pm 3$ & $34 \pm 2$ & $35 \pm 3$ & $34 \pm 3$ & $33 \pm 3$ \\
\hline GR & $35 \pm 2$ & $33 \pm 2$ & $34 \pm 3$ & $34 \pm 3$ & $34 \pm 2$ & $34 \pm 2$ & $34 \pm 3$ & $34 \pm 2$ & $34 \pm 3$ \\
\hline \multicolumn{10}{|c|}{ VM (l/min) } \\
\hline GL & $2,8 \pm 1$ & $2,4 \pm 1^{\mathrm{a}}$ & $2,4 \pm 1^{\mathrm{a}}$ & $2,4 \pm 1^{\mathrm{a}}$ & $2,2 \pm 1^{\mathrm{a}}$ & $2,2 \pm 1^{\mathrm{a}}$ & $2,9 \pm 1$ & $3,4 \pm 0,2$ & $3,4 \pm 1$ \\
\hline GXL & $3,5 \pm 1$ & $2,9 \pm 1^{\mathrm{a}}$ & $2,3 \pm 1^{\mathrm{a}}$ & $2,3 \pm 0,5^{\mathrm{a}}$ & $2,8 \pm 1^{\mathrm{a}}$ & $2,7 \pm 0,4^{\mathrm{a}}$ & $3,2 \pm 1$ & $3,4 \pm 0,6$ & $2,7 \pm 0,8$ \\
\hline GR & $3,8 \pm 1$ & $4,3 \pm 1^{b}$ & $4 \pm 2^{\mathrm{b}}$ & $4 \pm 2^{b}$ & $4,7 \pm 1^{b}$ & $4,3 \pm 2^{\mathrm{b}}$ & $3,7 \pm 1$ & $3,6 \pm 1$ & $3,6 \pm 1$ \\
\hline \multicolumn{10}{|c|}{$f(\mathrm{mov} / \mathrm{min})$} \\
\hline GL & $14 \pm 1$ & $13 \pm 1^{\mathrm{a}}$ & $12 \pm 1$ & $12 \pm 1$ & $12 \pm 1^{\mathrm{a}}$ & $12 \pm 1^{\mathrm{ab}}$ & $13 \pm 1^{\mathrm{ab}}$ & $13 \pm 1^{\mathrm{ab}}$ & $13 \pm 2^{\mathrm{ab}}$ \\
\hline GXL & $12 \pm 1$ & $12 \pm 2^{\mathrm{a}}$ & $12 \pm 1$ & $11 \pm 1$ & $11 \pm 2^{\mathrm{a}}$ & $11 \pm 2^{\mathrm{a}}$ & $11 \pm 2^{\mathrm{a}}$ & $11 \pm 1^{\mathrm{a}}$ & $10 \pm 1^{\mathrm{a}}$ \\
\hline GR & $15 \pm 1$ & $15 \pm 1^{\mathrm{b}}$ & $15 \pm 2$ & $13 \pm 3$ & $14 \pm 4^{\mathrm{b}}$ & $14 \pm 4^{b}$ & $14 \pm 3^{b}$ & $15 \pm 2^{b}$ & $16 \pm 2^{b}$ \\
\hline \multicolumn{10}{|c|}{$\mathrm{T}\left({ }^{\circ} \mathrm{C}\right)$} \\
\hline GL & $38,8 \pm 0,1$ & $38,7 \pm 0,1$ & $38,6 \pm 0,1$ & $38,4 \pm 17$ & $38,3 \pm 0,1$ & $38,2 \pm 0,1$ & $38,1 \pm 0,2$ & $38,1 \pm 0,2$ & $37,8 \pm 0,2$ \\
\hline GXL & $38,5 \pm 0,5$ & $38,4 \pm 1$ & $38,4 \pm 0,5$ & $38,2 \pm 1$ & $38,2 \pm 1$ & $38 \pm 1$ & $38 \pm 1$ & $37,9 \pm 1$ & $37,9 \pm 1$ \\
\hline \multirow[t]{2}{*}{ GR } & $38,8 \pm 0,2$ & $38,7 \pm 0,1$ & $38,6 \pm 0,1$ & $38,5 \pm 0,2$ & $38,4 \pm 0,2$ & $38,3 \pm 0,3$ & $38,3 \pm 0,3$ & $38,2 \pm 0,4$ & $38,2 \pm 0,4$ \\
\hline & & $135 \mathrm{~min}$ & $150 \mathrm{~min}$ & $165 \mathrm{~min}$ & $180 \mathrm{~min}$ & $195 \mathrm{~min}$ & $210 \mathrm{~min}$ & $235 \mathrm{~min}$ & $240 \mathrm{~min}$ \\
\hline \multicolumn{10}{|c|}{ FC (bat/min) } \\
\hline GL & & ---- & ---- & ---- & ---- & ---- & ---- & ---- & ---- \\
\hline GXL & & $79 \pm 8^{\mathrm{B}}$ & $74 \pm 12^{\mathrm{B}}$ & $80 \pm 10^{\mathrm{B}}$ & $72 \pm 8^{\mathrm{B}}$ & $76 \pm 6^{\mathrm{B}}$ & $81 \pm 10^{\mathrm{B}}$ & $82 \pm 10^{\mathrm{B}}$ & $78 \pm 7^{\mathrm{B}}$ \\
\hline GR & & $81 \pm 15^{\mathrm{AB}}$ & $74 \pm 6^{\mathrm{B}}$ & $72 \pm 6^{\mathrm{AB}}$ & $78 \pm 8^{\mathrm{AB}}$ & $74 \pm 4^{\mathrm{AB}}$ & $80 \pm 12^{\mathrm{AB}}$ & $76 \pm 20^{\mathrm{AB}}$ & $82 \pm 10^{\mathrm{AB}}$ \\
\hline \multicolumn{10}{|c|}{ PAS (mmHg) } \\
\hline GL & & ---- & ---- & ---- & ---- & ---- & ---- & ---- & ---- \\
\hline GXL & & $118 \pm 4$ & $120 \pm 9$ & $121 \pm 7$ & $119 \pm 7$ & $120 \pm 7$ & $120 \pm 4$ & $120 \pm 9$ & $118 \pm 9$ \\
\hline GR & & $110 \pm 13$ & $111 \pm 9$ & $110 \pm 2$ & $111 \pm 12$ & $110 \pm 11$ & $112 \pm 9$ & $120 \pm 6$ & $120 \pm 6$ \\
\hline \multicolumn{10}{|c|}{$\mathrm{ETCO}_{2}(\mathrm{mmHg})$} \\
\hline GL & & ---- & ---- & ---- & ---- & ---- & ---- & ---- & ---- \\
\hline GXL & & $36 \pm 3$ & $35 \pm 5$ & $35 \pm 4$ & $34 \pm 2$ & $35 \pm 3$ & $34 \pm 2$ & $34 \pm 2$ & $34 \pm 3$ \\
\hline GR & & $34 \pm 2$ & $35 \pm 2$ & $34 \pm 2$ & $34 \pm 2$ & $34 \pm 1$ & $34 \pm 1$ & $34 \pm 3$ & $33 \pm 3$ \\
\hline \multicolumn{10}{|c|}{ VM (l/min) } \\
\hline GL & & ---- & ---- & ---- & ---- & ---- & ---- & ---- & ---- \\
\hline GXL & & $2,7 \pm 1$ & $3 \pm 1$ & $3,1 \pm 1$ & $2,6 \pm 1$ & $2,8 \pm 1$ & $3,3 \pm 1$ & $3,9 \pm 1$ & $3,1 \pm 1$ \\
\hline GR & & $3,6 \pm 1$ & $3,3 \pm 1$ & $3,7 \pm 1$ & $3,2 \pm 1$ & $3,7 \pm 1$ & $3,8 \pm 1$ & $3,3 \pm 1$ & $3 \pm 1$ \\
\hline \multicolumn{10}{|c|}{$f(\mathrm{mov} / \mathrm{min})$} \\
\hline GL & & ---- & ---- & ---- & ---- & ---- & ---- & ---- & ---- \\
\hline GXL & & $11 \pm 1^{\mathrm{a}}$ & $10 \pm 1^{\mathrm{a}}$ & $10 \pm 1^{\mathrm{a}}$ & $10 \pm 1^{\mathrm{a}}$ & $11 \pm 1^{\mathrm{a}}$ & $11 \pm 1^{\mathrm{a}}$ & $11 \pm 1$ & $12 \pm 1$ \\
\hline GR & & $14 \pm 2^{b}$ & $13 \pm 2^{b}$ & $14 \pm 1^{\mathrm{b}}$ & $14 \pm 1^{\mathrm{b}}$ & $14 \pm 2^{b}$ & $13 \pm 2^{\mathrm{b}}$ & $13 \pm 1$ & $14 \pm 1$ \\
\hline \multicolumn{10}{|l|}{$\mathrm{T}\left({ }^{\circ} \mathrm{C}\right)$} \\
\hline GL & & ---- & ---- & ---- & ---- & ---- & ---- & ---- & ---- \\
\hline GXL & & $37,8 \pm 1$ & $37,8 \pm 1$ & $37,7 \pm 1$ & $37,7 \pm 1$ & $37,5 \pm 1$ & $37,5 \pm 1$ & $37,4 \pm 0,4$ & $37,4 \pm 0,4$ \\
\hline \multirow[t]{2}{*}{ GR } & & $38,1 \pm 0,4$ & $38 \pm 0,45$ & $38 \pm 0,4$ & $37,8 \pm 0,4$ & $37,8 \pm 0,4$ & $37,7 \pm 0,4$ & $37,6 \pm 0,3$ & $37,6 \pm 0,4$ \\
\hline & & Regressão & & & & \multicolumn{4}{|c|}{ Coeficiente de determinação $\left(r^{2}\right)$} \\
\hline \multicolumn{2}{|l|}{ GL } & \multicolumn{4}{|c|}{ temperatura $=38,8-0,0078$ tempo } & & & & \\
\hline GXL & & temperatura & $38,0-0,00$ & tempo & & & & & \\
\hline GR & & temperatura & $38,7-0,00$ & tempo & & & & & \\
\hline
\end{tabular}

Dentro de cada grupo, momentos seguidos de letras maiúsculas diferentes nas medianas (linha) diferem estatisticamente. Diferenças entre os grupos em cada momento estão expressas em letras minúsculas diferentes (coluna). $\mathrm{P}<0,05$. 
Tabela 2 - Mediana e amplitude semiquartílica do tempo até o relaxamento do esfíncter anal, perda e retorno do reflexo interdigital e tempo até a recuperação da posição quadrupedal. Valores médios do somatório das vértebras dessensibilizadas e localização anatômica do último espaço intervertebral dessensibilizado (no sentido caudo-cranial), com avaliação a partir do espaço lombo-sacro, em cães submetidos à tranqüilização com acepromazina e à anestesia epidural com lidocaína $2 \%$ com vasoconstritor (GL), xilazina 2\% associada à lidocaína (GXL) ou ropivacaína 1\% (GR).

\begin{tabular}{|c|c|c|c|c|c|c|}
\hline Grupos & $\begin{array}{l}\text { Esfíncter } \\
\text { (seg) }\end{array}$ & $\begin{array}{l}\text { Perda ID } \\
\text { (min) }\end{array}$ & $\begin{array}{l}\text { Retorno ID } \\
\text { (min) }\end{array}$ & $\begin{array}{l}\text { Quadrupedal } \\
\text { (min) }\end{array}$ & $\begin{array}{c}\mathrm{n}^{\circ} \text { vértebras } \\
\text { dessensibilizadas }\end{array}$ & Altura max.bloqueio \\
\hline GL & $30 \pm 0^{\mathrm{a}}$ & $1 \pm 0^{\mathrm{a}}$ & $120 \pm 5^{\mathrm{a}}$ & $130 \pm 9^{a}$ & $2,4^{\mathrm{a}}$ & L4 \\
\hline GXL & $30 \pm 15^{\mathrm{a}}$ & $1 \pm 1^{\mathrm{ab}}$ & $240 \pm 18^{b}$ & $248 \pm 10^{b}$ & $7,4^{\mathrm{b}}$ & $\mathrm{T} 11$ \\
\hline GR & $60 \pm 10^{b}$ & $2 \pm 1^{b}$ & $250 \pm 10^{b}$ & $263 \pm 13^{b}$ & $2^{\mathrm{a}}$ & L4 \\
\hline
\end{tabular}

Diferenças entre os grupos em cada momento estão expressas em letras minúsculas diferentes (coluna). P<0,05.

adversos decorrentes do uso de ropivacaína (MARKHAM \& FAULDS, 1996; PITHER et al., 2003).

Embora haja relatos de que a ação $(\alpha 2-$ adrenoceptora agonista da xilazina possa causar bradipnéia (KELAWALA et al., 1996), neste estudo, todos os parâmetros respiratórios apresentaram-se estáveis em todos os grupos. Sabe-se que, ao contrário dos anestésicos voláteis, que causam depressão cardiorrespiratória dose-dependente, normalmente a anestesia epidural não produz esses efeitos (MASSONE, 2003). Os maiores valores de volume-minuto observados no GR, dificilmente, podem ser explicados por um efeito farmacológico e, possivelmente, devem estar relacionados tanto com um maior valor basal, quanto com a variação biológica.

Em estudo realizado em bovinos, REHAGE et al. (1994) demonstraram que a administração epidural de $0,05 \mathrm{mg} \mathrm{kg}^{-1}$ de xilazina produziu efeito sedativo moderado, fato similar ao observado neste estudo, em cães. Tal efeito parece ser positivo no sentido de manter os animais calmos durante o período anestésico.

Tabela 3 - Valores individuais da extensão máxima do bloqueio sensitivo, perante o estímulo nociceptivo mecânico (teste do panículo), em cães submetidos à tranqüilização com acepromazina e à anestesia epidural com lidocaína 2\% com vasoconstritor (GL), xilazina $2 \%$ associada à lidocaína (GXL) ou ropivacaína $1 \%(\mathrm{GR})$.

\begin{tabular}{lllcll}
\hline GL & Panículo & GXL & Panículo & GR & Panículo \\
\hline 1 & L6 & 1 & T11 & 1 & L5 \\
2 & L5-L6 & 2 & T12 & 2 & L5-L6 \\
3 & L4 & 3 & L1 & 3 & L4 \\
4 & L4 & 4 & T11 & 4 & L6 \\
5 & L4-L5 & 5 & L1 & 5 & L4-L5 \\
6 & L4 & 6 & L1 & 6 & L5-L6 \\
7 & L6 & 7 & T12 & 7 & L5 \\
8 & L4 & 8 & T12 & 8 & L5 \\
9 & L5 & 9 & T12 & 9 & L5 \\
10 & L4 & 10 & L1 & 10 & L6 \\
\hline
\end{tabular}

A discreta redução da temperatura retal no decorrer do tempo pode ser atribuída à ação vasodilatadora e depressora dos mecanismos termorreguladores do hipotálamo induzida pela acepromazina(HALL \& CLARKE, 1991). Adicionalmente, a xilazina também atua deprimindo o centro de termorregulação (KLIDE et al., 1975). Pode-se sugerir que a perda progressiva de calor seja, provavelmente, decorrente do efeito desses fármacos sobre o centro termorregulador, acrescido do miorrelaxamento produzido. $\mathrm{O}$ aquecimento foi realizado somente com aquecedor ambiente, que parece não ter sido suficiente para minimizar a perda de calor. Apesar da aparente redução de temperatura, especialmente no GL, nenhum animal apresentou hipotermia, com a mínima temperatura registrada de $37,8^{\circ} \mathrm{C}$, não havendo, portanto, relevante importância biológica, neste achado.

O período de latência observado com a lidocaína epidural, neste estudo, corroborou resultados previamente relatados em cães (ISHIY et al., 2002). No homem, o período de latência após a administração epidural de ropivacaína variou entre 5 a 10 minutos (PITHER et al., 2003). A duração do bloqueio obtido com lidocaína foi semelhante à relatada por SKARDA (1987) e ISHIY et al. (2002), porém mais prolongado que o observado por CRUZ et al. (1997). A duração anestésica da associação de xilazina e lidocaína por via epidural foi semelhante à relatada por RECTOR et al. (1997). A adição da xilazina à lidocaína proporciona períodos mais prolongados de ação em relação ao uso isolado do anestésico local. Em pôneis, a administração de xilazina epidural permitiu analgesia mais pronunciada e de duração mais longa em relação à lidocaína, em doses e volumes equipotentes (FIKES et al., 1989). A xilazina apresenta efeito anestésico local, caracterizado pelo bloqueio no potencial de ação e na velocidade de condução (AZIS \& MARTIN, 1978). Ademais, o efeito analgésico da xilazina também é conferido pela ativação dos alfa2- 
adrenoceptores na medula espinhal, a qual confere período de ação prolongado de analgesia (BRANSON et al., 1993).

O bloqueio mais cranial, proporcionado pela associação de xilazina e lidocaína, em comparação ao uso isolado de anestésicos locais, provavelmente, pode viabilizar a realização de procedimentos cirúrgicos abdominais craniais, como a OSH, visto que a inervação ovariana se faz pela emergência do $3^{\circ}$ e do $4^{\circ}$ nervos lombares (BAILEY et al., 1988). A xilazina proporciona analgesia moderada, com miorrelaxamento satisfatório(GROSS, 2003); porém, convém ressaltar que o uso da xilazina diluída somente em água destilada não promoveu miorrelaxamento em cães em estudo piloto, observando-se relaxamento muscular somente quando associada à lidocaína.

\section{CONCLUSÕES}

Diante das condições experimentais deste estudo, pode-se concluir que a utilização de lidocaína, xilazina/lidocaína ou ropivacaína por via epidural produz anestesia na região retroumbilical. A associação de xilazina/lidocaína causa bloqueio anestésico mais cranial, atingindo a região entre T11-L1, o que favorecerá a realização de cirurgias pré-umbilicais. No entanto, deve-se ressaltar que o uso de xilazina/ lidocaína deve ser reservado para animais hígidos, uma vez que a bradicardia foi detectada em todos os animais anestesiados por essa associação farmacológica.

\section{FONTES DE AQUISIÇÃO}

${ }^{\mathrm{a}}$ Acepran $0,2 \%$, Univet.

${ }^{b}$ Xylocaína 2\% com 1:200.000 de Adrenalina, Astra.

'Naropin $10 \mathrm{mg} / \mathrm{ml}$, Astra.

${ }^{\mathrm{d}}$ Rompum, Bayer.

${ }^{e}$ Monitor PC SCOUT, Space Labs Medical

${ }^{\mathrm{f}}$ Módulo de Expansão de Pressão Arterial Não-Invasiva, Space Labs Medical.

${ }^{g}$ Monitor PC SCOUT, Space Labs Medical.

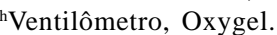

iMT-500-1, Microtherm.

jFoley Catheter 22fr e 20cc com válvula, Bard.

\section{COMITÊ DE ÉTICA}

Este estudo foi aprovado pelo Comitê de Ética da instituição de origem, sob o protocolo n.21/99.

\section{REFERÊNCIAS}

AZIZ, M.A.; MARTIN, R.J. Alpha agonist and local anaesthetic properties of xylazine. Zentralblatt für Veterinärmedizin, Reihe A, v.25, n.3, p.181-188, 1978.

BAILEY, C.S. et al. Spinal nerve root origins of the cutaneous nerves of the canine pelvic limb. American Journal of Veterinary Research, v.49, n.1, p.115-119, 1988.
BRANSON, K.R. et al. Duration of analgesia induced by epidurally administered morphine and medetomidine in dogs. Journal of Veterinary Pharmacology and Therapeutics, v.16, n.3, p.369-372, 1993.

CRUZ, M.L. et al. Epidural anaesthesia using lignocaine, bupivacaine or a mixture of lignocaine and bupivacaine in dogs. Journal of Veterinary Anaesthesia, v.24, n.1, p.30-32, 1997.

FELDMAN, H.S. et al. Antinociceptive and motor-blocking efficacy of ropivacaine and bupivacaine after epidural administration in the dog. Regional Anesthesia, v.21, n.4, p.318-326, 1996.

FIKES, L.W. et al. A preliminary comparison of lidocaine and xylazine as epidural analgesics in ponies. Veterinary Surgery, v.18, n.1, p.85-86, 1989.

GROSS, M.E. Tranquilizantes, agonistas (2.-adrenérgicos e agentes relacionados. In: ADAMS, H.R. Farmacologia e terapêutica em veterinária. 8.ed. Rio de Janeiro: Guanabara Koogan, 2003. Cap.14, p.249-284.

HALL, L.W.; CLARKE, K.W. Principles of sedation, analgesia and premedication. Veterinary Anaesthesia. 9.ed. Londres: Bailiière Tindall, 1991. Cap.4, p.51-79.

ISHIY, H.M. et al. Uso da lidocaína isolada ou associada à quetamina ou ao butorfanol, em anestesia epidural em cadelas submetidas à ovariosalpingohisterectomia. Revista Brasileira de Ciência Veterinária, v.9, n.1, p.134-136, 2002.

KELAWALA, N.H. et al. Effects of epidural xylazine with diazepam premedication in dogs. Indian Veterinary Journal, v.73, n.5, p.552-557, 1996.

KLIDE, A.M. et al. Cardiopulmonary effects of xylazine in dogs. American Journal of Veterinary Research, v.36, p.931-935, 1975.

LUNA, S.P.L. et al. Effects of four anaesthetic protocols on the neurological and cardiorespiratory variables of puppies born by caesarean section. Veterinary Record, 27, v.154, n.13, p.387-389, 2004.

MARKHAM, A.; FAULDS, D. Ropivacaine - A review of its pharmacology and therapeutic use in regional anaesthesia. Drugs, v.52, p.429-449, 1996.

MASSONE, F. Anestesia local. In: Anestesiologia veterinária: farmacologia e técnicas. 4.ed. Rio de Janeiro: Guanabara Koogan, 2003. Cap.3, p.33-42.

MAZZANTI, G.; BRAGHIROLI, L. Analgesic antiinflammatory action of Pfaffia paniculata (Martius) Kuntze. Phytotherapy Research, v.8, p.413-416, 1994.

MCMURPHY R.M. Postoperative epidural analgesia. Veterinary Clinics of North America: Small Animal Practice, v.23, n.4, p.703-716, 1993.

PITHER, C.E. et al. A comparison of the dynamics and pharmacokinetics of ropivacaine $7.5 \mathrm{mg} / \mathrm{ml}$ with and without epinephrine used for epidural anaesthesia in urological surgery. Clinical Drug Investigation, v.23, n.4, p.245-253, 2003. 
RECTOR, E. et al. Beurteilung der antinozizeptiven wirkung von xylazin nach epiduraler applikation bei hunden in allgemeinanästhesie mit isofluran. Berliner und Munchener Tierarztliche Wochenschrift, v.110, n.1, p.15-23, 1997.

REHAGE, J. et al. Use of xylazine for epidural anaesthesia in cattle. Deutsche Tierarztliche Wonchenschrift, v.101, p.14-16, 1994.

SAHNI, Y.P. et al. Involvement of mediators of pain in analgesic activity of Withania somnifera. Indian Veterinary Journal, v.72, p.1035-1039, 1995.
SKARDA, R.T. Local and regional analgesia. In: SHORT, C.E. Principles and practice of veterinary anesthesia. Los Angeles: Williams \& Wilkins, 1987. p.91-134.

VIEIRA, R.J. et al. Xilazina e lidocaína: anestesia peridural comparativa em bovinos. Veterinária e Zootecnia, v.5, p.105-112, 1993.

WEISSMAN, C. The metabolic response to stress An overview and update. Anesthesiology, v.73, p.308-327, 1990.

WOLFF, A.P. et al. Extradural ropivacaine and bupivacaine in hip surgery. British Journal of Anaesthesia, v.74, n.4, p.458-460, 1995. 